\title{
NON-LINEAR RING MODEL CALIBRATION WITH FREQUENCY ANALYSIS OF BETATRON OSCILLATIONS
}

\author{
R. Bartolini ${ }^{1}$, F. Schmidt ${ }^{2}$, \\ ${ }^{1}$ Diamond Light Source Ltd, Rutherford Appleton Laboratory, Chilton, Oxon, OX11 0QX, UK. \\ ${ }^{2}$ CERN, CH-1211, Geneva 23, Switzerland.
}

\begin{abstract}
A precise model of an accelerator ring is crucial to achieve ultimate performance both in synchrotron light sources and high energy synchrotrons. Algorithms have been developed to calibrate the linear model of the ring. They have been successfully applied experimentally to determine and correct the linear optics of the machine. More recently the Frequency Map Analysis has been used to model also the non-linear optics. We propose here a technique based on the fit of non-linear spectral lines to recover the non-linear driving terms and to compensate the non-linear field errors around the ring.
\end{abstract}

\section{INTRODUCTION}

Recent developments in the modelling of the accelerator lattice have proven very useful to correct and improve the performance of synchrotron light sources [1, 2] and high energy synchrotrons [3]. A well established technique is based on the analysis of the orbit response matrix to model or correct the linear optic of the machine. The LOCO algorithm [1] and equivalent packages have been used to correct the linear optic of various synchrotrons, including restoring the periodicity of the ring and linear coupling compensation. Another well known technique is based on the Frequency Map Analysis (FMA) [4] of the betatron oscillations and has been applied successfully at ALS [5] and more recently at Bessy-II [6]. This technique gives information about the non-linear behaviour of the ring and allows to test or control the non-linear part of the model.

The frequency analysis of betatron motion can also be used to extract information on the resonance driving term that affect the non-linear dynamics of the particle beam. It has been shown [7] that each spectral line is proportional to a specific resonance driving term, at least in the first order of perturbation theory with the multipole gradients. In this article we show that the measurement of the spectral lines can be used to correct the non-linearities and to generate a model of the ring that reproduces the dynamics. This method is based on a $\chi^{2}$ fit of lattice element gradient to minimize the difference between the spectral content of the measured model and the ideal model. The spectral lines whose amplitude and phase should be controlled are selected according to which resonance appears to be most excited and the lattice parameters should be selected among those that excited the corresponding resonance, e.g. sextupole gradients error can be fitted by controlling spectral lines excited by third order resonances.

Lastly, we will give an example of application of these techniques to analysis tracking data of a realistic model of the DIAMOND [8] lattice and we show that this method has beneficial effects on the dynamic aperture (DA) of the ring.

\section{THE METHOD}

According to the first order perturbative theory of nonlinear betatron motion, the beam oscillation in the horizontal plane can be written in normalized coordinates, as

$$
\begin{aligned}
& \hat{x}(n)-i \hat{p}_{x}(n)=\sqrt{2 I_{x}} e^{i\left(2 \pi v_{x} N+\psi_{x 0}\right)} \\
& -2 i \sum_{j k l m} j f_{j k l m}\left(2 I_{x}\right)^{\frac{j+k-1}{2}}\left(2 I_{y}\right)^{\frac{l+m}{2}} . \\
& \cdot e^{i\left[(1-j+k)\left(2 \pi v_{x} N+\psi_{x 0}\right)+(m-l)\left(2 \pi v_{y} N+\psi_{y 0}\right)\right]}
\end{aligned}
$$

where $I_{x}$ and $I_{y}$ are the horizontal and vertical action, $\psi_{x 0}$ and $\psi_{\mathrm{y} 0}$ are the horizontal and vertical initial phases, $v_{\mathrm{x}}$ and $v_{y}$ are the horizontal and vertical betatron tunes and the coefficients $f_{j k l m}$ are the generating function terms. An analogous expression is valid for the vertical plane. These coefficients are related to the Hamiltonian coefficients $\mathrm{h}_{\mathrm{jklm}}$ according to

$$
f_{j k l m}=\frac{h_{j k l m}}{1-e^{2 \pi i\left[(j-k) v_{x}+(l-m) v_{y}\right]}}
$$

which are in turn proportional to the distribution of magnetic elements along the ring, e.g. the coefficients $\mathrm{h}_{3000}, \mathrm{~h}_{1020}, \mathrm{~h}_{1002}$ are all proportional to the sextupoles strength and are responsible for the excitation of the third order resonances $(3,0),(1,2),(1,-2)$ respectively. The coefficients $f_{j k l m}$ vary along the ring and their value at each BPM can be obtained from the spectral decomposition of the betatron oscillation signal using an enhanced FFT algorithm such as those developed in the NAFF [9] or SUSSIX [10] codes.

For a particular resonance that needs to be compensated or that significantly differs from the s-dependence pattern expected from the model, one can build a target vector $\mathrm{A}_{\text {meas }}$ whose components are the amplitude and phase of the spectral lines computed at each BPM. 
These coefficients will depend on the value of the particular magnetic elements that excite the corresponding resonances. If the resonance $(3,0)$ is targeted, then the target vector to use is given by the amplitude and phase of the spectral line $(-2,0)$ which is excited by the sextupoles in the ring:

$$
\bar{A}_{\text {meas }}^{(-2,0)}=\left(A_{1}, \ldots, A_{\text {Nbpm }} ; \phi_{1}, \ldots, \phi_{\text {Nbpm }}\right)
$$

where Nbpm is the total number of BPMs in the ring. Each component of this vector will depend on the particular distribution of sextupoles in the ring, therefore we can write

$$
\bar{A}_{\text {meas }}^{(-2,0)}=\bar{A}_{\text {meas }}^{(-2,0)}\left(S_{1}, \ldots, S_{\text {Nsext }}\right)
$$

where $S_{k}$ is the strength of the k-th sextupoles and Nsext is the total number of sextupoles.

This target vector is then compared with the same target vector computed from the ideal model $\mathrm{A}_{\text {model }}$. The distance between these two vectors

$$
\chi^{2}=\sum_{j=1}^{2 N b p m}\left(A_{\text {model }}^{(-2,0)}(j)-A_{\text {meas }}^{(-2,0)}(j)\right)
$$

gives a measure of the discrepancy between the real accelerator and the model. This quantity can be minimized by a least square minimization procedure that involves fitting the strength of the magnetic element directly responsible for the excitation of that particular spectral line. To do so one has to built a sensitivity matrix that provides the jacobian of the dependence of the target vector $\mathrm{A}$ on the fit parameter chosen

$$
M_{j k}=\frac{\partial A_{j}}{\partial S_{k}}
$$

The values of the fit parameter can be obtained inverting the relation

$$
\bar{A}_{\text {meas }}=\bar{A}_{\text {model }}+\bar{M} \cdot \bar{S}
$$

to

$$
\bar{S}=\bar{M}^{-1} \cdot\left(\bar{A}_{\text {meas }}-\bar{A}_{\text {model }}\right)
$$

Since the matrix $M$ will not be square in general, it can be inverted by means of an SVD decomposition. Furthermore the dependence of the target vector A with the fit parameter S is non-linear, therefore the SVD needs to be iterated until convergence is reached.

The target vector A can be built using the amplitude and or the phase of several spectral lines according to the particular problem and the particular set of magnetic errors that we wish to control in the lattice. Notice that this method can be used also to control the linear part of the ring and to compensate $\beta$-beating and coupling [11]. With this respect, the outlined method can give complementary information to those obtained by a LOCO-like analysis of the linear lattice, the latter being based on closed orbit measurements rather than excited betatron oscillation measurements. At the same time it can provide information on the non-linear part of the ring model, giving complementary information to those obtained by the FMA, by looking at the whole spectral content of the betatron oscillations computed at all BPMs, rather than the tune dependence with amplitude computed at one BPM. Of course the simple relation between spectral line and resonance driving term (i.e. non-linear magnet gradients) is based on the perturbative analysis valid to the first order in the element gradient and breaks down when higher order become effective.

\section{APPLICATION TO THE DIAMOND LATTICE}

We report the application of the method to the tracking data obtained from a realistic model of the DIAMOND lattice. The lattice is a 24 cell Double Bend Achromat (DBA). Each cell has 7 BPMs, 10 quadrupoles and 7 sextupoles: all quadrupoles and sextupoles have independent power supplies. The quadrupoles are arranged in 10 families and the sextupoles in 8 families.

As a first example we introduced random errors on a chromatic sextupole family including 24 sextupoles. The errors in the sextupole strength are assigned from a Gaussian distribution with r.m.s. equal to $5 \%$ of the average sextupole strength in the ring $\left(\sigma=0.25 \mathrm{~m}^{-2}\right)$, cut at three sigmas. The target vector used contains the amplitude of the spectral lines excited by the resonances $(3,0)$ and $(1,2)$, i.e.

$$
\bar{A}=\left(A_{1}^{(-2,0)}, \ldots, A_{\text {Nbpm }}^{(-2,0)}, A_{1}^{(0,2)}, \ldots, A_{\text {Nbpm }}^{(0,2)}\right)
$$

In Fig. 1 we report the error values assigned to 24 sextupoles and the reconstructed errors with the SVD procedure as outlined in the previous paragraph.

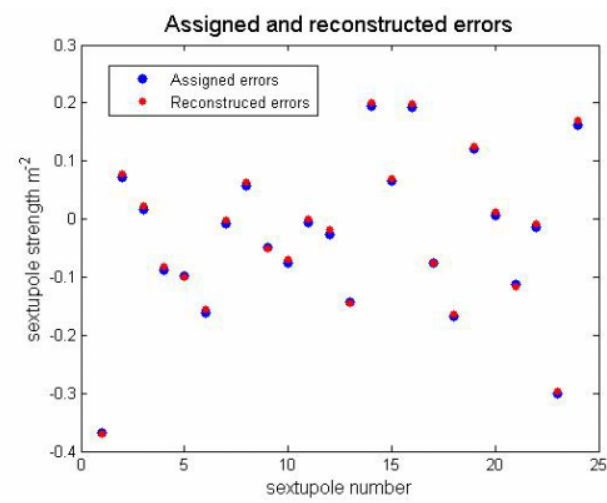

Fig. 1: Random errors assigned to 24 sextupoles and reconstructed values with the SVD procedure 
In this case the correction identified exactly all the errors assigned.

We then assigned random errors to all the 168 sextupoles in the ring. Using the same definition for the target vector we obtained the results shown in Fig. 2

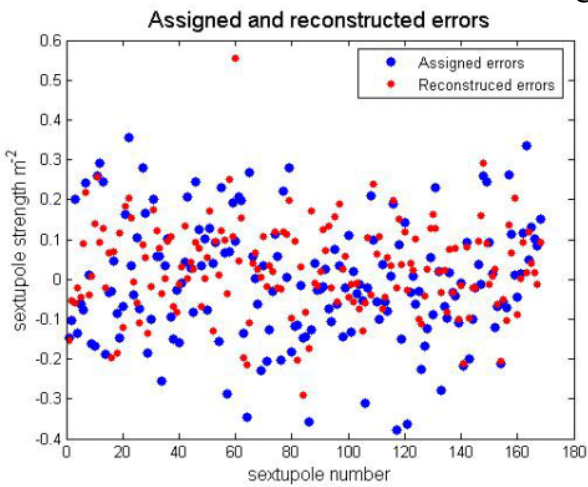

Fig. 2: Random errors assigned to 168 sextupoles and reconstructed values with the SVD procedure

It is evident that now the algorithm does not identify all the assigned errors one by one, however the new distribution of sextupole strengths is able to correct effectively the behaviour of the spectral lines used in the target vector as shown in Fig. 3.
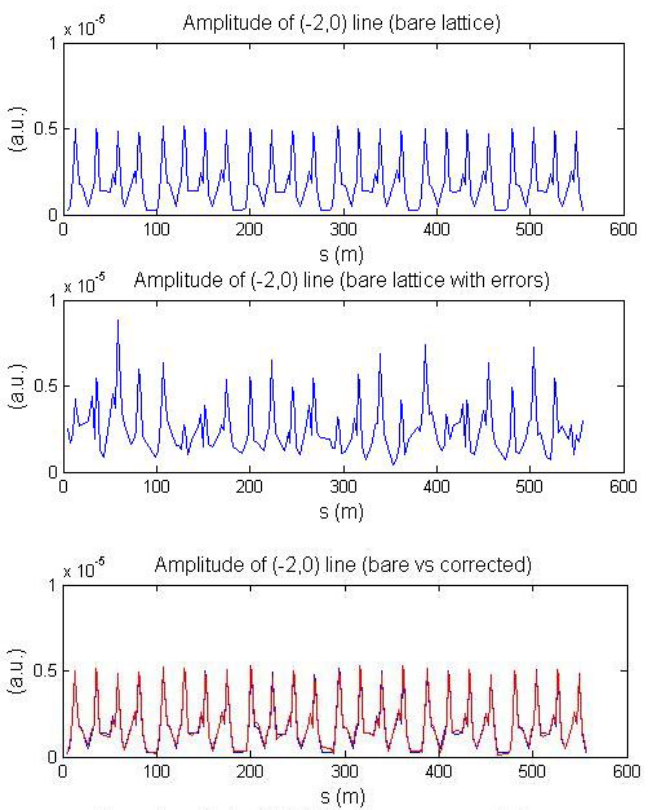

Fig. 3: Spectral lines $(-2,0)$ for the ideal lattice (top); for the lattice with added random errors in the sextupoles (middle); and (bottom) comparison of spectral lines $(-2,0)$ of the ideal (blue) and that lattice (red) with random errors and its corrections.

The correction has some benefit also on the onmomentum DA, as shown in Fig. 4. Before correction (top) the DA is $10 \mathrm{~mm}$ horizontally, $8 \mathrm{~mm}$ vertically, after correction (middle) it becomes $15 \mathrm{~mm}$ horizontally and $10 \mathrm{~mm}$ vertically. Furthermore the diffusion rate indicated by the colour in the two graphs show that globally the corrected machine is more stable. The DA is not restored to the values of the bare lattice (bottom), indicating that the correction of a limited number of the spectral line is not necessarily fully correlated with the DA.
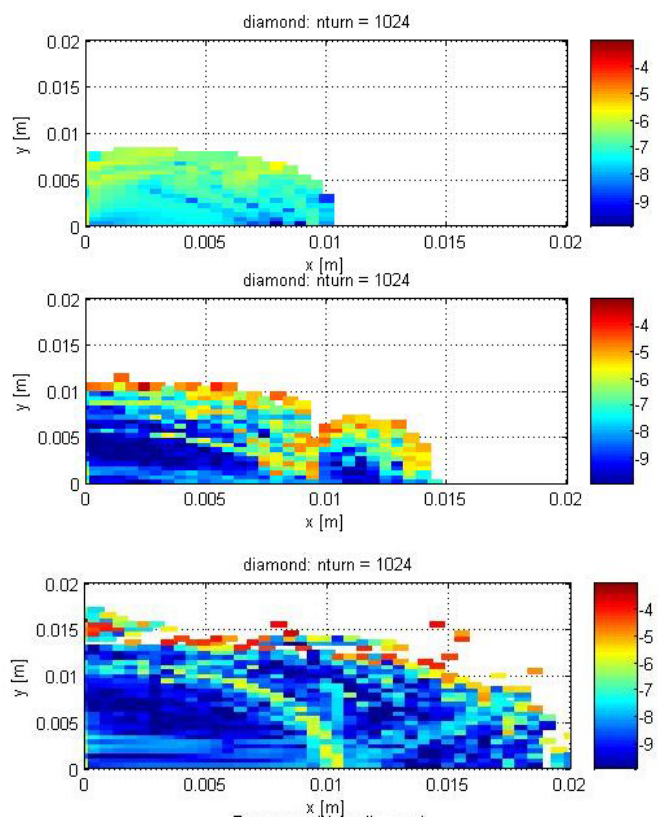

Fig. 4: DA (on-momentum) before correction (top), after correction (middle) of the sextupoles and for the ideal lattice (bottom).

\section{CONCLUSIONS}

We presented a new technique for the analysis of the nonlinear part of the accelerator model based on an SVD fit of the spectral lines at all BPMs. The method looks promising and is effective in the tracking simulation of the Diamond model. Its effectiveness in dealing with real machine data still has to be proven: noisy data, BPM gains and decoherence of kicked beam oscillations will predictably limit the applicability of this technique. However, the method can be used in conjunction with LOCO and FMA to help building a realistic model of the ring, and give further insight in the behaviour of the linear and non linear dynamics of the beam.

\section{REFERENCES}

[1] J. Safranek, NIM A388, 27, (1997).

[2] D. Robin et al., EPAC 2000, 136, (2000); C. Steier et al., EPAC 2000, 1077, (2000); Y. Papaphilippou et al., PAC 2003, 3189, (2000).

[3] G. Hoffstaetter et al., EPAC 2002, 407, (2002).

[4] L. Nadolski and J. Laskar, Phys. Rev. E, 407, (2002).

[5] C. Steier et al., Phys. Rev. E, 407, (2002).

[6] P. Kuske, this proceedings.

[7] R. Bartolini and F. Schmidt, Part. Acc., 59, 93, (1998)

[8] DIAMOND Synchrotron Light Source. Report of the design specifications, "The Green Book", March 2002.

[9] J. Laskar et al., Physica D, 56, 253, (1992).

[10] R. Bartolini and F. Schmidt, CERN-SL note 98-017 (AP), 1999.

[11] F. Zimmermann et al., SLAC-AP 113, (1998). 